\title{
Interactions in dye-microcavity photon condensates and the prospects for their observation
}

\author{
R. A. Nyman* \\ Centre for Cold Matter, Blackett Laboratory, Imperial College London, Prince Consort Road, SW7 2BW, United Kingdom \\ M. H. Szymańska ${ }^{\dagger}$ \\ Department of Physics, University of Warwick, Coventry CV4 7AL, United Kingdom
}

(Received 20 December 2013; published 24 March 2014)

\begin{abstract}
We derive the equation of motion for a Bose-Einstein condensate of photons in a dye-microcavity system, starting from Maxwell's equations. Our theory takes into account mirror shape, Kerr-type intensity-dependent refractive index and incoherent pumping and loss. The resulting equation is remarkably similar to the GrossPitaevskii equation for exciton-polariton condensates, despite the different microscopic origins. We calculate the incoherent photoluminescence spectrum of the photon condensate, which shows the Bogoliubov-type excitations around the mean field at thermal equilibrium. Both open- and closed-system models are presented to account for, respectively dissipation and inhomogeneities. Considering realistic parameters and experimental resolution, we estimate that by observing the angle-resolved spectrum of incoherent photoluminescence it is possible to resolve dimensionless interaction parameters of order $10^{-5}$, two orders of magnitude below current estimates. Thus we expect that this technique will lead to accurate measurements of the interactions in photon condensates.
\end{abstract}

DOI: 10.1103/PhysRevA.89.033844

PACS number(s): 42.50.Ct, 03.75.Hh, 67.90.+z

\section{INTRODUCTION}

Bose-Einstein condensation is usually thought of as a lowtemperature phenomenon. However, Klaers et al. made the first room-temperature Bose-Einstein condensate (BEC) [1] by confining photons in a cavity filled with fluorescent dye to provide the photons with an effective mass and to allow photon thermalization at fixed photon number. Under well-chosen experimental conditions [2,3], despite the continuous drive and dissipation, the photons come into thermal equilibrium with a dye, and the evidence for the Bose-Einstein condensation transition was strong. Due to the room-temperature operation [4] and relatively simple experimental setup in comparison to other lower-temperature condensates this system offers now an ideal playground to study macroscopic quantum systems.

While Bose-Einstein condensation was initially proposed in the context of noninteracting Bose gas, the interactions that make this phase transition experimentally possible lead to a plethora of collective quantum phenomena. For example, bosons with repulsive interactions, such as liquid helium- 4 and atomic gases, make superfluids while attractive interactions lead to pairing of fermions and superconductivity, superfluidity of helium-3, and molecular BECs in trapped gases. The interactions in exciton-polariton condensates [5] play a crucial role in the observed excitation spectra [6,7] and superfluid behavior such as quantized vortices [8] and persistent currents $[9,10]$. Dye-microcavity photon BECs share some of the features of exciton polaritons, such as the dissipation, pumping and loss. Unlike exciton polaritons, photon BECs are in the weak light-matter coupling regime, and are also thought to be very close to thermal equilibrium. A superfluid description of light, setting aside the thermalization process, was first proposed by

\footnotetext{
*Corresponding author: r.nyman @imperial.ac.uk

†Present address: Department of Physics and Astronomy, University College London, Gower Street, London WC1E 6BT, United Kingdom.
}

R. Chiao [11-13], which follows on from earlier descriptions of inhomogeneous gain media in optical cavities [14-16]. The suggestion was to use dilute vapours of alkali metals as a nonlinear medium, together with an optical resonator to constrain the dispersion relation of the light.

In photon BEC, the photon-photon interactions have not yet been accurately measured, and even a full theoretical description is still lacking. Two mechanisms for interactions have been suggested: (i) the Kerr effect of intensity-dependent refractive index, and (ii) a temperature-dependent solvent refractive index, with temperature inhomogeneities driven by inhomogeneities of the light. Preliminary measurements indicated a dimensionless two-dimensional (2D) interaction parameter of about $(7 \pm 3) \times 10^{-4}$, which is consistent with the second mechanism. However, this mechanism does not act at the single-particle level and so does not have any effect on, for example, short-range particle correlations. This thermal mechanism is also slow compared to experimental time scales, typically taking about $1 \mathrm{~ms}$ to act, compared to a $1 \mu \mathrm{s}$ pump pulse duration. The Kerr effect happens on time scales as fast as the spontaneous emission lifetime of the dye, of the order of $1 \mathrm{~ns}$. The strength of Kerr interactions is unknown, however, expectations that it would be tiny have already motivated theoretical studies on how the phase coherence typical of BECs builds up even in the complete absence of particle-particle interactions [17].

In this work, we derive an equation of motion for the pumped photon condensate in a dissipative microcavity, complete with effects of Kerr-type intensity-dependent refractive index, which leads to an effective photon-photon interaction. We use the equivalence of this equation to complex GrossPitaevskii equations (cGPEs) used for other systems to help obtain the excitation spectrum and incoherent photoluminescence (IPL), the light that leaks through the cavity mirrors, including the limited experimental resolution. We argue that the IPL spectrum is an excellent diagnostic for the photon-photon interaction strength. We propose an experimental apparatus for measuring the angle-resolved photoluminescence spectrum to 
an accuracy sufficient to determine interaction strength even as much as two orders of magnitude more precisely then current estimates. This method is only sensitive to the (fast) Kerr effect and not the (slow) thermal lensing. Measurement and understanding of the microscopic origin of interactions in photon BEC are a prerequisite for any possible superfluid effects to be seen in experiments.

\section{EQUATION OF MOTION FOR A PHOTON BEC}

The equation of motion for the condensate wave function of a photon BEC in a dye-filled microcavity can be derived starting from Maxwell's equations in a nonlinear dielectric medium. The closest equivalent theory concerns excitonpolariton condensates, where the light-matter coupling is strong, and therefore the matter component must be treated quantum mechanically. Our Maxwell's equation approach is valid only for photon BEC, where the light-matter coupling is weak. The cavity is so short that only one longitudinal mode is relevant.

We propagate the wave from one mirror of the cavity to the other and back again. The net change in electric field over one cycle of propagation divided by the time that cycle takes determines the time derivative for the electric field. Note, that it is also possible to obtain a similar equation of motion by considering a decomposition over quasinormal modes of the optical resonator in appropriate paraxial and slowly varying envelope approximations [18].

\section{A. Nonlinear wave propagation}

The nonlinear electric polarizability of the medium can be accounted for by writing the electric polarization as a linear part plus a nonlinear part [19]: $\mathbf{P}=\mathbf{P}_{L}+\mathbf{P}_{N L}$. The constitutive relation is $\mathbf{D}=\epsilon_{0} \epsilon_{L} \mathbf{E}+\mathbf{P}_{N L}$, where the linear permittivity (in the limit of low-intensity light) is $\epsilon_{L}=n_{L}^{2}=$ $1+\chi_{L}$ and $\chi_{L}$ is the linear susceptibility. We define $n_{L}$ as the refractive index at low intensity, and the wave equation for the electric field becomes:

$$
\nabla(\nabla \cdot \mathbf{E})-\nabla^{2} \mathbf{E}+\frac{n_{L}^{2}}{c^{2}} \ddot{\mathbf{E}}=-\frac{1}{c^{2}} \ddot{\mathbf{P}}_{N L}
$$

with the dot representing the time derivative. We make the paraxial approximation and assume that the electric field can be written as a scalar (constant polarization throughout space, perpendicular to the direction of propagation). We consider a traveling wave solution, $E=E_{0}(x, y, z) e^{i\left(k_{L} z-\omega t\right)}$ with a slowly varying envelope: $z$ is the axis of the optical resonator, as shown in Fig. 1.

For annotation's sake, where only one argument of $E_{0}$ is given, it is $z ; x$ and $y$ are left implicit. With two arguments, they are $x$ and $y$, leaving $z=0$ implicit. The angular frequency and wave number of the light are $\omega$ and $k_{L}=\omega n_{L} / c$ respectively. Now, using conventional definitions for the Kerr-type nonlinearity, the paraxial wave equation becomes:

$$
-\left\{2 i k \frac{\partial E_{0}}{\partial z}+\frac{\partial^{2} E_{0}}{\partial z^{2}}+\nabla_{\perp}^{2} E_{0}\right\}=\frac{k_{L}^{2}}{n_{L}^{2}} 3 \chi^{(3)}\left|E_{0}\right|^{2} E_{0},
$$

where $\nabla_{\perp}^{2}=\frac{\partial^{2}}{\partial x^{2}}+\frac{\partial^{2}}{\partial y^{2}}$. Starting from the electric field at $z=$ 0 , we can find the field some small propagation distance away
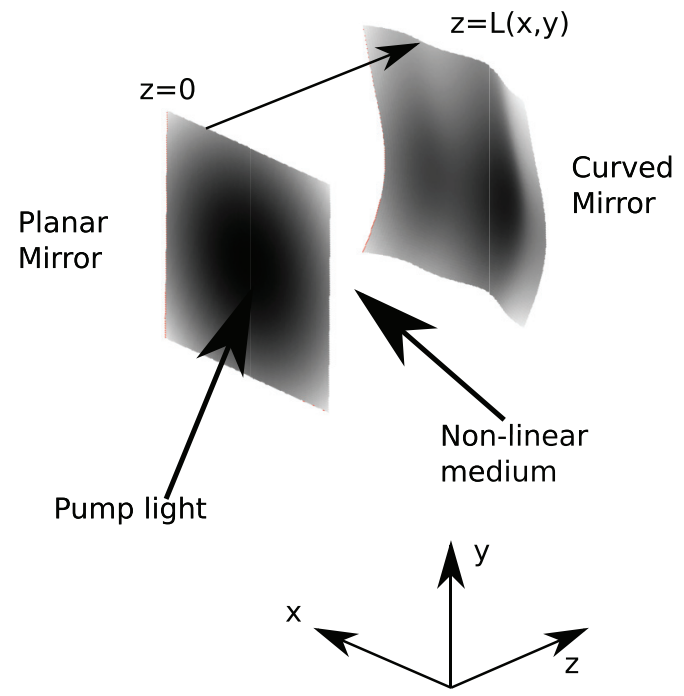

FIG. 1. A cavity filled with a nonlinear medium, which allows the photons to come to thermal equilibrium faster than they leave the cavity. The coordinate system is as used in the rest of this paper. The length variations of the cavity play the role of a potential energy landscape for the photons. The cavity is so short that only one longitudinal mode is excited, and individual photons have a dispersion relation equivalent to that of massive particles. Light that leaks through the cavity mirrors, photoluminescence, can be observed using standard optics such as cameras and spectrometers.

at $z=L$ using a first-order Taylor expansion:

$E_{0}(L) \simeq E_{0}(0)-\frac{L}{2 i k_{L}}\left\{\nabla_{\perp}^{2} E_{0}(0)+\frac{k_{L}^{2}}{n_{L}^{2}} 3 \chi^{(3)}\left|E_{0}(0)\right|^{2} E_{0}(0)\right\}$.

\section{B. To and fro between mirrors}

We now consider a forward traveling wave with an electric field envelope $E_{0} \rightarrow(x, y, 0)$ and use Eq. (3) to find the electric field at the other mirror. We will allow the mirror surface position to vary, so the cavity length is a function of position: $L(x, y)=L_{0}+\delta L(x, y)$. For length variations that are much less than the wavelength of the light, we make a linear approximation for the phase of the light, and neglect additional envelope propagation effects. The forward propagating field at the second mirror is

$$
\begin{aligned}
E_{0}^{\rightarrow}(x, y, L(x, y))= & E_{0}^{\rightarrow}(0)\left(e^{-i k_{L} L_{0}}+i k_{L} \delta L\right) \\
& -\frac{L_{0}}{2 \mathrm{i} k_{L}} e^{i k_{L} L_{0}}\left\{\nabla_{\perp}^{2} E_{0}^{\rightarrow}(0)\right. \\
& \left.+\frac{k_{L}^{2}}{n_{L}^{2}} 3 \chi^{(3)}\left|E_{0}^{\rightarrow}(0)\right|^{2} E_{0}^{\rightarrow}(0)\right\} .
\end{aligned}
$$

The mirrors have transmission and reflectance $\tau$ and $r$ respectively. After reflection, the backwards propagating field at $L(x, y)$ is $E_{0}^{\leftarrow}(L(x, y))=-r E_{0}^{\rightarrow}(L(x, y))$. Propagation to the first mirror at $z=0$ and subsequent reflection follow the same pattern. Finally, to complete a cycle, inhomogeneous pump light enters through the mirror at $z=0$. In order to model saturable, incoherent pumping, it is sufficient to write 
the pump term in the form $\left(\alpha-\beta\left|E_{0}\right|^{2}\right) E_{0}$. Here, $\alpha$ represents difference between gain via stimulated scattering of photons into the condensate and cavity mirror loss, and $\beta$ governs saturation [20]. $\beta$ depends on the pump intensity and position, and can be derived for given experimental conditions in the steady-state limit.

The time taken for a cycle is given by $\delta t=c / 2 n_{L} L_{0}$, where $c$ is the speed of light in vacuum. The electric field change $\delta E_{0}$ in one cycle is derived as above, and then we convert finite differences to derivatives: $\delta E_{0} / \delta t=\frac{\partial E_{0}}{\partial t}=\dot{E}_{0}$. Highorder terms in small quantities are neglected. The equation of motion for the electric field envelope at the first mirror, assuming that it varies slowly compared to the cavity roundtrip time becomes:

$$
\begin{aligned}
\frac{1}{i \omega} \frac{\partial E_{0}}{\partial t}= & {\left[\left(\frac{\delta L}{L_{0}}-\frac{\delta \omega}{\omega}\right)+\frac{1}{k_{L}^{2}} \nabla_{\perp}^{2}+\frac{3 \chi^{(3)}}{2 n_{L}^{2}}\left|E_{0}\right|^{2}\right] E_{0} } \\
& -\frac{i}{2 q \pi}\left[\left(\alpha-\beta\left|E_{0}\right|^{2}\right)-(1-r)\right] E_{0},
\end{aligned}
$$

where $\chi^{(3)}$ is the Kerr nonlinearity; $\delta \omega$ the cavity detuning; $k_{L}=2 \pi q / L_{0}=\omega n_{L} / c ; n_{L}$ the refractive index in the limit of low light intensity; $q$ the longitudinal mode number. Only the lowest relevant order in small quantities is retained. Because $E_{0}$ is a classical field in this equation, i.e., the mean-field part, thermal or quantum fluctuations, the noncondensed photons, are not described by this equation.

Finally, it is straightforward to include the effects of inhomogeneous linear refractive index (which appears as an increase in the effective length of the cavity), or spatially variable mirror reflectivities.

\section{Similarity to a complex GP equation}

A typical form for the cGPE for the condensate wave function $\psi$ in a system with incoherent pumping (as in photon $\mathrm{BEC})$ is:

$$
-i \hbar \frac{\partial \psi}{\partial t}=\left[V(\mathbf{r})-\frac{\hbar^{2}}{2 m} \nabla_{\perp}^{2}+g|\psi|^{2}+i\left(\gamma_{\text {net }}-\Gamma|\psi|^{2}\right)\right] \psi
$$

where $\gamma_{\text {net }}$ is the difference between the pump rate and cavity decay rate and is equal to $\alpha$ in Eq. (5), and $\Gamma$ describes the saturation of pumping (ensuring stability). In the steady state $\Gamma=\gamma_{\text {net }} /|\psi(0,0)|^{2}$. In two dimensions $\int d x d y|\psi(x, y)|^{2}=$ $N_{\mathrm{BEC}}$, where $N_{\mathrm{BEC}}$ is the number of particles in the condensate.

Comparing (5) and (6) the first term in Eq. (5) is equivalent to potential energy in the cGPE with an additional energy offset due to the detuning between the cavity mode and the light. The second term comes from diffraction of the light, and corresponds to kinetic energy, while the third term to the interactions. The energy stored in the electric field of the standing wave in the cavity is $\frac{1}{2} n_{L}^{2} L_{0} \epsilon_{0} \int d x d y\left|E_{0}(x, y)\right|^{2}=$ $N_{\text {BEC }} \hbar \omega$. We can define the quantity $m$ through $\hbar \omega=m c^{2} / n_{L}^{2}$, and this will play the role of an effective photon mass. With these analogies, we can convert the equation of motion for $E_{0}$ electric field Eq. (5) into the cGPE Eq. (6) with the steady-state mean-field solution $\psi=\psi_{0} e^{-i \mu t / \hbar}$ where $\mu$ is the chemical potential:

$$
\begin{gathered}
\psi_{0}=E_{0} \sqrt{\frac{n_{L}^{2} \epsilon_{0} L_{0}}{2 \hbar \omega}} \\
g=\frac{3 \hbar^{2} \omega^{2}}{n_{L}^{4} \epsilon_{0} L_{0}} \chi^{(3)}=\frac{\hbar^{2}}{m} \tilde{g},
\end{gathered}
$$

where $\tilde{g}$ is the dimensionless $2 \mathrm{D}$ interaction parameter [21].

\section{EXCITATION SPECTRUM AND PHOTOLUMINESCENCE}

The IPL, which can be measured by angle- and energyresolved techniques, for a system in thermal equilibrium is given by the Bose-Einstein occupation function $n_{B}(\omega)$ times the spectral weight $[22,23]$ :

$$
P_{L}(\mathbf{k}, \omega)=n_{B}(\omega) W(\mathbf{k}, \omega) .
$$

The spectral weight can be obtained from the retarded Green's function for the response of the system to perturbations, $G_{R}$ :

$$
W(\mathbf{k}, \omega)=2 \operatorname{Im}\left[G_{R}(\mathbf{k}, \omega)\right],
$$

as shown for example in Ref. [24]. The exact form of $G_{R}$ depends on the model used and here we determine $G_{R}$ for a dissipative and driven case given by Eq. (6) (the open system model) as well as for a simplified case where the dissipative terms are neglected (the closed system model). The two models give almost identical IPL since the decay processes in a photon BEC are very small. Thus we further use the closed-system model to determine the influence of the trapping potential on IPL. We note that thermal-lensing-type interactions do not affect the excitation spectrum, as they occur on timescales much longer than thermal fluctuations and quasiparticle lifetimes.

\section{A. Open-system model to deal with dissipation}

In the homogeneous case, $V(x, y)=0$, the steady-state mean-field solution of Eq. (6) $\psi_{0}$, is found, by writing a solution with time variation $e^{-i \mu t / \hbar}$ to obtain the chemical potential $\mu=g\left|\psi_{0}\right|^{2}$. Note that in the Thomas-Fermi limit of a harmonic trap with frequency $\Omega_{0}$, the chemical potential is $\hbar \Omega_{0} \sqrt{\frac{\tilde{g} N_{\mathrm{BEC}}}{\pi}}$. The equation of motion for small (linear) variations about this mean field, $\delta \psi$ can be obtained by starting from a total wave function as $\psi=\psi_{0}+\delta \psi$ and subtracting the mean-field solution.

Comparison with the Hermitian conjugate leads to a system of linear equations in $\delta \psi$ and $\delta \psi^{*}$. The matrix operator that relates the two is the inverse Green's function [22], $(\mathcal{G})^{-1}$, which is also known as the Bogoliubov operator [25]:

$$
-i \hbar \frac{\partial}{\partial t}\left(\begin{array}{c}
\delta \psi \\
\delta \psi^{*}
\end{array}\right)=(\mathcal{G})^{-1}\left(\begin{array}{c}
\delta \psi \\
\delta \psi^{*}
\end{array}\right) .
$$

The relevant component for the photoluminescence is the diagonal $(\delta \psi, \delta \psi)$ component of the full Green's function: $G_{R}(\mathbf{k}, \omega)=\mathcal{G}^{11}(\mathbf{k}, \omega)$.

In the stationary and homogeneous case, the inversion is most easily performed in Fourier space 
to give [7]:

$$
\begin{aligned}
& \mathcal{G}_{\mathcal{R}}(\mathbf{k}, \omega) \\
& =\frac{1}{\hbar^{2} \omega\left(\omega+2 \mathrm{i} \gamma_{\mathrm{net}}\right)-\epsilon_{\mathbf{k}}\left(\epsilon_{\mathbf{k}}+2 \mu\right)} \\
& \quad \times\left(\begin{array}{cc}
\mu+\epsilon_{\mathbf{k}}+\hbar \omega+i \hbar \gamma_{\mathrm{net}} & -\mu+i \hbar \gamma_{\mathrm{net}} \\
-\mu-i \hbar \gamma_{\mathrm{net}} & \mu+\epsilon_{\mathbf{k}}-\hbar \omega+i \hbar \gamma_{\mathrm{net}}
\end{array}\right),
\end{aligned}
$$

where $\epsilon_{\mathbf{k}}=\frac{\hbar^{2} k^{2}}{2 m}$ is the kinetic energy of a free particle, and $\mathbf{k}$ is in the $x, y$ plane.

The IPL from the dye-microcavity sample at temperature $T$ using the open-system model of Eq. (6) but assuming that photons thermalize is

$$
P_{L}^{\text {(open) }}(\mathbf{k}, \omega)=\frac{4 \hbar}{e^{\frac{\hbar \omega}{k_{B} T}}-1} \frac{\gamma_{\text {net }}\left(\epsilon_{\mathbf{k}}+\omega\right)\left(\epsilon_{\mathbf{k}}+2 \mu+\omega\right)}{4 \gamma_{\text {net }}^{2} \omega^{2}+\left(\epsilon_{\mathbf{k}}^{2}+2 \epsilon_{\mathbf{k}} \mu-\omega^{2}\right)^{2}} .
$$

The energy scale is relative to the chemical potential, so $\mu$ does not appear in distribution factor. We note that this expression is approximate. We have assumed a thermal distribution of fluctuations (noncondensed photons). For full self-consistency, a frequency-dependent distribution of fluctuations would imply frequency-dependent decay and pump rates, which, for simplicity, we have approximated as being frequency independent. However, only small corrections in the relevant region around the poles would come about from considering non-Markovian decay rates.

Figure 2 shows the IPL energy-momentum spectrum, in which the Bogoliubov dispersion is clearly visible. The parameters of the calculation are experimentally achievable, and similar to values in Ref. [1]. For values of $\tilde{g}$ above about $10^{-5}$, the difference between free particles and photon quasiparticle excitations from the interacting condensate is very clear.

\section{B. Closed-system model to deal with inhomogeneities}

By ignoring the dissipative term in Eq. (6) we determine the IPL for the closed system. In the homogeneous case, the difference between the open- and closed-system models is mostly notable at low momentum and energy. The closed system follows the usual Bogoliubov modes down to zero momentum but the open-system modes become diffusive at very low momenta [25-27]. However, the effects of interactions are observable in the IPL energy-momentum spectrum at moderate momenta and energy, and so the two models largely agree for the purposes of this work.

Photon BEC, however, is not homogeneous. In the local density approximation (LDA), we proceed by using a local chemical potential with $\mu^{\prime}(\mathbf{r})=\mu-V(\mathbf{r})$. The energy spectrum for excitations is $\xi_{\mathbf{k}}(\mathbf{r})=\sqrt{\epsilon_{\mathbf{k}}\left(\epsilon_{\mathbf{k}}+2 \mu^{\prime}(\mathbf{r})\right)}$. This is a local version of the Bogoliubov spectrum [28]. There are finite temperature corrections [29] which we are neglecting here. The local spectral weight is then given by:

$$
\begin{aligned}
W^{(\text {closed })}(\mathbf{k}, \omega ; \mathbf{r})= & \frac{\epsilon_{\mathbf{k}}+\mu^{\prime}(\mathbf{r})+\xi_{\mathbf{k}}(\mathbf{r})}{2 \xi_{\mathbf{k}}(\mathbf{r})} \delta\left(\hbar \omega-\xi_{\mathbf{k}}(\mathbf{r})\right) \\
& -\frac{\epsilon_{\mathbf{k}}+\mu^{\prime}(\mathbf{r})-\xi_{\mathbf{k}}(\mathbf{r})}{2 \xi_{\mathbf{k}}(\mathbf{r})} \delta\left(\hbar \omega+\xi_{\mathbf{k}}(\mathbf{r})\right) .
\end{aligned}
$$
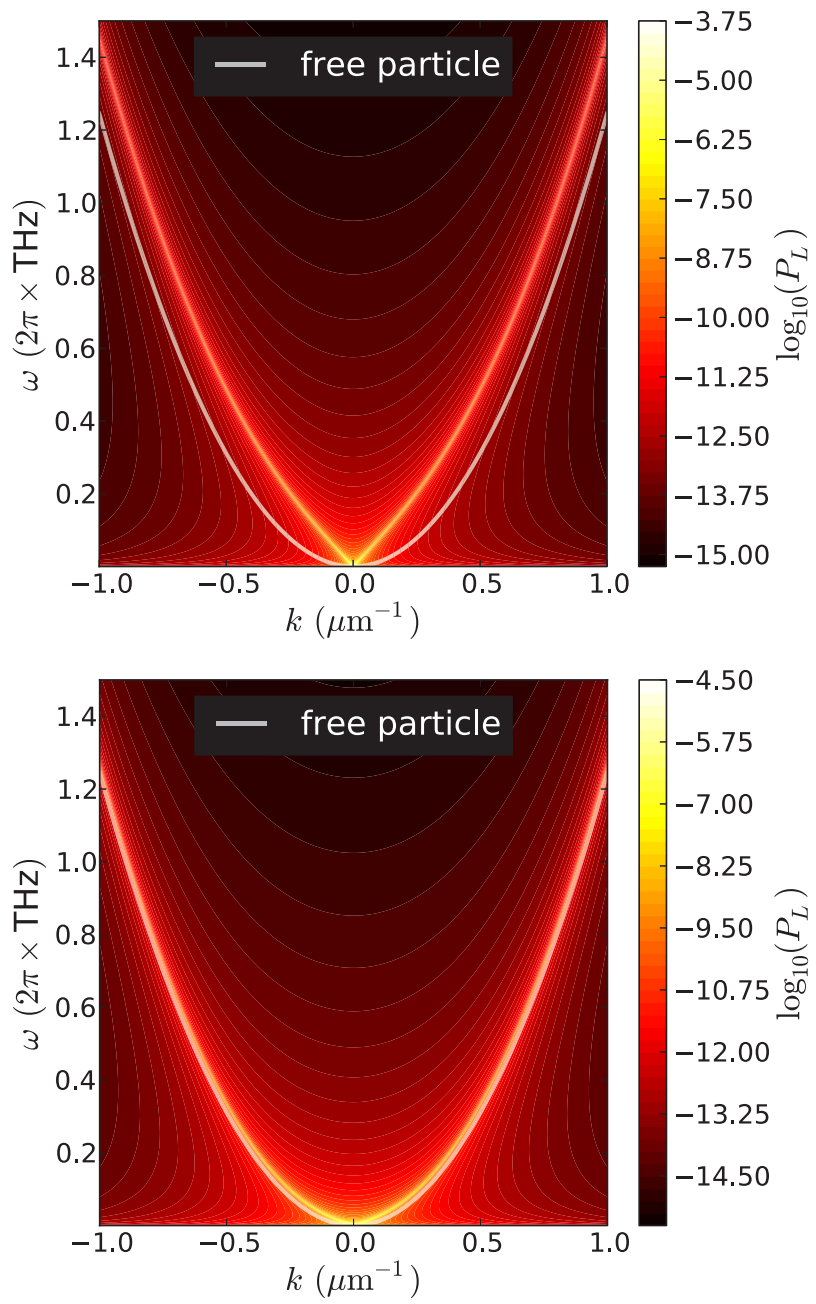

FIG. 2. (Color online) Examples of photoluminescence, calculated using the open-system model. On this scale, the closed system results look the same. Top: dimensionless interaction parameter $\tilde{g}=10^{-3}$ with $10^{5}$ condensed photons. Bottom: $\tilde{g}=10^{-5}$ and $10^{5}$ condensed photons. As a guide for eye we plot the dispersion relation for free particle with the same photon mass. Angular frequency $\omega$ is taken relative to the chemical potential. Other parameters: $\gamma_{\text {net }}=2 \pi \times 1 \mathrm{GHz} ; T=300 \mathrm{~K}$; central density calculated from Thomas-Fermi profile of photons in a circularly-symmetric harmonic trap of frequency $40 \mathrm{GHz}$.

The energy scale is shifted such that excitations of energy $\hbar \omega=0$ are at the chemical potential. Some broadening is put into the system by hand by adding an imaginary part $\hbar \kappa$ to the energy, $\epsilon \rightarrow \epsilon-i \hbar \kappa$ and, when integrated around the zero of the argument, $\delta(\epsilon) \rightarrow \frac{1}{\pi} \frac{\hbar \kappa}{\epsilon^{2}+\hbar^{2} \kappa^{2}}$. The local Bose occupation factor becomes $n_{B}(\omega ; \mathbf{r})=\frac{\hbar}{e^{[\hbar \omega-V(\mathbf{r})] / k_{B} T}-1}$ where $n_{B}(\omega)=\iint d^{2} \mathbf{r} n_{B}(\omega ; \mathbf{r}) / A$ and $A$ is a typical area of the system, e.g., $2 \pi \mu / m \Omega_{0}^{2}$ for in the Thomas-Fermi limit in a harmonic potential. The total photoluminescence observable becomes:

$$
P_{L}^{\text {(closed) }}(\mathbf{k}, \omega)=\iint d^{2} \mathbf{r} n_{B}(\omega ; \mathbf{r}) W^{\text {(closed) }}(\mathbf{k}, \omega ; \mathbf{r}) .
$$

An inhomogeneous confining potential means that the density varies across the condensate, which in turn leads to variations 
in the Bogoliubov spectrum, i.e., the speed of sound. The integration over all positions of the condensate causes the lines in the IPL energy-momentum spectrum to broaden, making it more difficult to see the effects of interactions. The photoluminescence calculated here is the incoherent part; coherent photoluminescence will be emitted from the condensate mode. It may contain a large range of momenta, but it will all be at the lowest energy available, and so incoherent and coherent light can easily be distinguished (condensate broadening in energy is expected to be very small on the scale of Fig. 2).

\section{ENERGY-MOMENTUM SPECTROSCOPY}

It is possible to observe the photoluminescence resolved in both energy and one component of momentum. The angleresolved photoluminescence spectrum (ARPLS) of excitonpolariton samples has been successfully measured, demonstrating the effect of interactions on the Bogoliubov dispersion relation [6,7]. The basic experimental optical apparatus to be used for photon BEC ARPLS is shown in Fig. 3.

The angle of a photon relative to the optic axis inside the cavity is $\boldsymbol{\theta}_{\text {int }}=\left(\theta_{x}, \theta_{y}\right)=\arctan \left(\mathbf{k} / k_{0}\right)$ with $k_{0}=q \pi n_{L} / L_{0}$ being the typical wave number. The angle that the emitted light makes to the optic axis is then $\theta_{\text {ext }}=\arcsin \left(n_{L} \sin \theta_{\text {int }}\right)$. The photoluminescence is at the focus of the objective lens of focal length $f_{\mathrm{obj}}$, and so the displacement from the optic axis is $(x, y)=n_{L} f_{\mathrm{obj}} \mathbf{k} / k_{0}$ (in the small-angle approximation). Behind the objective, the light passes through a slit of size $d_{\text {slit }}$ in the $y$ direction so only the $k_{y} \simeq 0$ components make it through. The image in momentum space is unaffected by subsequent optics. In order to improve spectral resolution, the image is magnified in the $y$ direction by a cylindrical

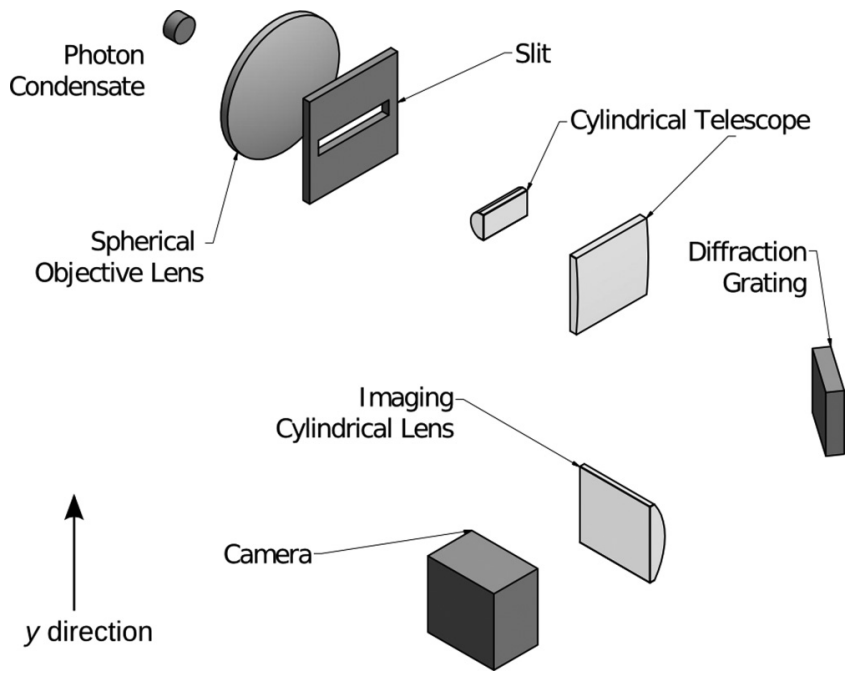

FIG. 3. A schematic diagram of angle-resolved photoluminescence spectroscopy (ARPLS). Photoluminescence from the photon condensate is collimated by the (spherical) objective lens. Components with wave numbers $k_{y} \simeq 0$ only pass through the slit. A cylindrical telescope magnifies the light in the $y$ direction, before it strikes a reflective diffraction grating, whose rules run parallel to the $x$ direction. The (cylindrical) imaging lens then ensures that the image on the camera corresponds to momentum $(x)$ and energy $(y)$. telescope of magnification $M_{y}$ and it strikes a reflective grating, whose lines are parallel to the $x$ direction and spaced by $d_{\text {grating }}$ in $y$. The first-order diffraction angle in the $y$ direction is $\theta_{y}=\arcsin \left(\lambda / d_{\text {grating }}\right)$, with $\lambda$ being the wavelength of light. The light passes through a cylindrical imaging lens of focal length $f_{\mathrm{im}}$, and the camera sits at this focus.

\section{A. Experimental limits to measuring interactions}

The mapping between $\left(k_{x}, \lambda\right)$ and position on screen is blurred by diffraction in the propagation of the light from source to detector. We analyze what the minimum resolvable momentum and energy would be, and what that means for the minimum resolvable interaction strength. In general, interactions will be detectable when the Bogoliubov spectrum is significantly different from the free-particle spectrum. This happens on energy scales less than $\xi_{\text {min }} \simeq 2 \mu$, and momentum scales less than $p_{\min } \simeq 2 \sqrt{m \mu}$.

For numerical evaluation, we need a value of the dissipation rate $\gamma_{\text {net }}$ of Eq. (6). The rate of scattering into the condensate is of the same order as the rate photons scatter from the dye, which depends on the dye concentration: $\gamma_{R}=1 / n_{\text {dye }} \sigma_{\text {dye }}\left(c / n_{L}\right)$ where $n_{\text {dye }} \simeq 10^{24}$ molecules $/ \mathrm{m}^{3}$, and is typically about $2 \pi \times 2-6 \mathrm{GHz}$ for Refs. [1,2]. An experimentally achievable cavity loss rate, $\kappa_{\mathrm{cav}} \simeq 2 \pi \times 1 \mathrm{GHz}$, and is governed by the mirror quality. Then, $\gamma_{\text {net }}=\gamma_{R}-\kappa_{\mathrm{cav}}$ is of order $2 \pi \times 1 \mathrm{GHz}$.

\section{Resolution in momentum}

The monochromator optics for energy resolution means that the Fourier-space image (after the objective lens and slit) will propagate and diffract before it reaches the camera. The propagation distance between objective lens and camera is $L_{\text {prop. }}$ A range of small transverse wave numbers $\delta_{k}$ corresponds to a region of size $n_{L} f_{\text {obj }} \delta_{k} / k_{0}$ at the objective, which will diffract to a region of size $\delta_{x}^{\text {(cam) }}=2 L_{\text {prop }} / n_{L} f_{\text {obj }} \delta_{k}$ at the camera (in the far field). Inverting this expression gives the diffraction limit for transverse wave number. Considering the mapping between angle and position, the equivalent limit set by the pixel size $\delta_{x}^{p x}$ of the camera is $\delta_{k} / k_{0}=\delta_{x}^{(p x)} / n_{L} f_{\mathrm{obj}}$. The optimum results will be achieved with diffraction limit roughly equal to pixelization limit, and we find:

$$
\delta_{k}^{(\min )}=\frac{2}{n_{L} f_{\mathrm{obj}}} \sqrt{\frac{\pi L_{\mathrm{prop}}}{\lambda}} .
$$

The same analysis yields an optimal pixel size of $\delta_{x}^{(p x)}=$ $\sqrt{L_{\text {prop }} \lambda / \pi}$. Putting in plausible experimental values $f_{\text {obj }}=$ $0.2 \mathrm{~m}, L_{\text {prop }}=0.3 \mathrm{~m}$ and $\lambda=580 \mathrm{~nm}$, we obtain a minimum in-plane momentum resolution of $1.3 \times 10^{4} \mathrm{~m}^{-1}$. The appropriate camera pixel size would be about $180 \mu \mathrm{m}$, which is trivially achievable. It is clearly advantageous to use a long focal length objective: to collimate a useful range of momenta, a lens with $f_{\text {obj }}=0.2 \mathrm{~m}$ should be $20 \mathrm{~mm}$ diameter. The equivalent size of the slit in momentum space should be no bigger than the expected momentum resolution of the entire optical system. In real space, that means that the slit should be about the same size as the detector pixels, $d_{\text {slit }} \sim 180 \mu \mathrm{m}$. 


\section{Resolution in energy}

Energy resolution is limited by the size of the beam at the grating. The resolving power for the first-order diffraction fringe is approximately equal to the number of grating lines covered by the incident beam: $\delta_{\lambda}=\lambda d_{\text {grating }} / D$. Reasonable experimental parameters are $1 / d_{\text {grating }}=900$ lines $/ \mathrm{mm}$ and $D=d_{\text {slit }} M_{y}=18 \mathrm{~mm}$ (implying a cylindrical telescope of magnification approximately 75). The resulting wavelength resolution is $\delta_{\lambda}=0.04 \mathrm{~nm}$, or equivalently $\delta_{\epsilon}=h \times 30 \mathrm{GHz}$ for $580 \mathrm{~nm}$ emission. With an imaging lens focal length of $f_{\mathrm{im}}=50 \mathrm{~mm}$, the detector pixel size required not to compromise this resolution is $4 \mu \mathrm{m}$ : a commonplace pixel size for a CCD camera.

\section{B. Minimum detectable interaction strength}

For reasonable parameters of $\Omega_{0}=2 \pi \times 40 \mathrm{GHz}$ and $N_{\text {BEC }}=10^{5}$, and optics as previously described, we find that it should be possible to resolve interactions as weak as $\tilde{g}_{\text {min }} \simeq\left(\frac{\hbar^{2} \delta_{k}^{2}}{4 m}\right)^{2} \frac{\pi}{N_{\mathrm{BEC}}\left(\hbar \Omega_{0}\right)^{2}}=2 \times 10^{-10}$ (if momentum is the limiting resolution) or $\tilde{g}_{\text {min }} \simeq \delta_{\epsilon}^{2} \frac{\pi}{N_{\mathrm{BEC}}\left(2 \hbar \Omega_{0}\right)^{2}}=2 \times 10^{-5}$ (if energy resolution is the limiting factor). It is worth noting that for extremely weak interactions, the Thomas-Fermi approximation used in deriving the photoluminescence spectrum is unlikely to be very accurate.

The implication for the experimenter is that the momentum is easily resolved, so most experimental effort will be required to attain the best possible energy resolution. Interactions 40 times weaker than those reported in Ref. [1] should be detectable. Advanced data analysis could further improve the sensitivity. An example of plausible experimental data is shown in Fig. 4, which includes both energy and momentum instrumental broadening and the effects of saturation and finite

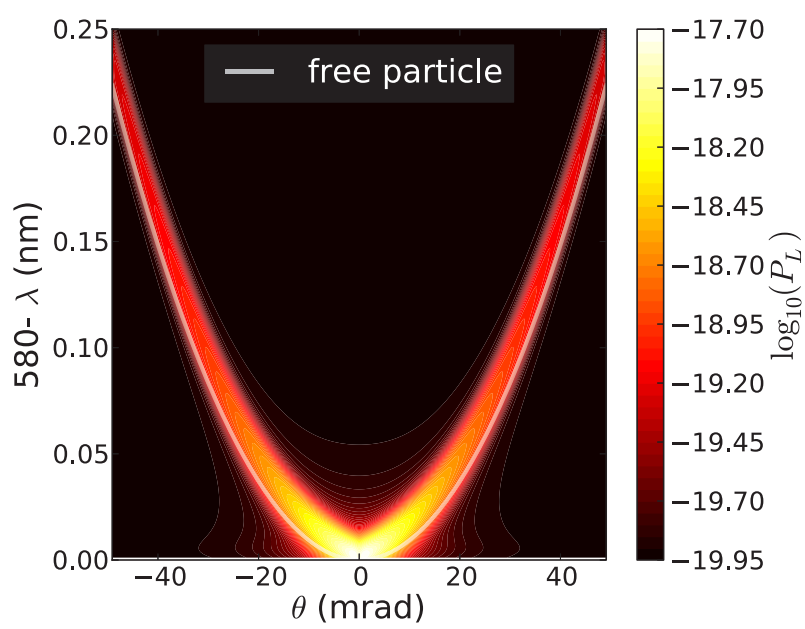

FIG. 4. (Color online) Observable photoluminescence energymomentum spectrum using a closed-system model, including the effects of the inhomogeneous confining potential, finite instrumental resolution in momentum and energy, and the finite dynamic range of a typical camera. Experimental parameters are described in the main text. Dimensionless interaction strength, number of condensed photons, temperature and trap frequency are $10^{-5}, 10^{5}, 300 \mathrm{~K}$, and $40 \mathrm{GHz}$ respectively. dynamic range of the detector camera (noise is not included in the model).

If the energy resolution required cannot be matched by grating spectroscopy, then an external Fabry-Perot cavity spectrometer would be a viable alternative. The minimum energy resolution in that case would most likely be set by the intrinsic linewidth of the resonator that contains the photon condensate, probably about $1 \mathrm{GHz}$. The limit on $\tilde{g}$ would in that case be somewhere around $10^{-6}$.

There is very little literature on the nonlinear susceptibility of dyes such as rhodamine. The closest available data is for very short pulses, which gives an underestimate of the nonlinearity since the steady-state excited-state population has not been reached. Taking a reasonable value for the scattering cross section of rhodamine [30] of $\sigma=2 \times 10^{-22} \mathrm{~m}^{2}$, and using the result of Ref. [31], we infer $\chi^{(3)} \simeq 5 \times 10^{-20}(\mathrm{~m} / \mathrm{V})^{2}$. This in turn implies a lower bound for the $2 \mathrm{D}$ dimensionless interaction parameter: $\tilde{g}>2 \times 10^{-7}$.

The intensity-dependent refractive index may come from an effect as simple as saturation of the excited-state population. For two-level systems, at short wavelengths one expects negative $\chi^{(3)}$ leading to repulsive interactions, but attractive interactions for long wavelengths. This frequency-dependent (i.e., also time-dependent) interaction strength leaves open the possibility for retarded interactions which will complicate the analysis of excitations about the condensate, and could lead to so-far unpredicted phenomena.

\section{CONCLUSIONS}

To conclude, we believe that the photoluminescence spectrum from a photon BEC can be observed using standard optical elements (lenses, a diffraction grating and a camera), with a sufficient resolution to detect dimensionless interaction parameters as small as about $10^{-5}$. The method measures only the fast, Kerr-type interactions and avoids the apparent interactions that come from the temperature-dependent refractive index of the solvent. If a Fabry-Perot resonator were used, the resolution may be an order of magnitude better. This compares well to the best available data in the literature on nonlinear susceptibilities in rhodamine dyes, and we can expect interaction effects in photon BECs to be experimentally observed via the energy-momentum spectrum. Knowledge of the magnitude and nature of the interactions in photon BEC is important for the observation of photon superfluidity. Likewise, applications in quantum metrology, i.e., optical measurement of fragile samples, depend on the photon-photon correlations, which are strongly affected by the microscopic nature of the interactions.

\section{ACKNOWLEDGMENTS}

We are grateful to EPSRC for funding this work (R.A.N. for funding from the fellowship EP/J017027/1 and M.H.S. for funding under fellowship EP/K003623/1 and Grant No. EP/I028900/1). We thank Jan Klaers, Jonathan Keeling, and Peter Kirton for informative discussions. 
[1] J. Klaers, J. Schmitt, F. Vewinger, and M. Weitz, Nature (London) 468, 545 (2010).

[2] J. Klaers, F. Vewinger, and M. Weitz, Nature Phys. 6, 512 (2010).

[3] P. Kirton and J. Keeling, Phys. Rev. Lett. 111, 100404 (2013).

[4] J. D. Plumhof, T. Stöferle, L. Mai, U. Scherf, and R. F. Mahrt, Nat. Mater. 13, 247 (2014).

[5] J. Kasprzak, M. Richard, S. Kundermann, A. Baas, P. Jeambrun, J. Keeling, M. FM Marchetti et al., Nature (London) 443, 409 (2006).

[6] S. Utsunomiya, L. Tian, G. Roumpos, C. Lai, N. Kumada, T. Fujisawa, M. Kuwata-Gonokami, A. Löffler, S. Höfling, A. Forchel et al., Nature Phys. 4, 700 (2008).

[7] G. Roumpos, M. Lohse, W. H. Nitsche, J. Keeling, M. H. Szymańska, P. B. Littlewood, A. Löffler, S. Höfling, L. Worschech, A. Forchel, and Y. Yamamoto, Proc. Natl. Acad. Sci. 109, 6467 (2012).

[8] K. G. Lagoudakis, M. Wouters, M. Richard, A. Baas, I. Carusotto, R. André, L. S. Dang, B. Deveaud-Plédran et al., Nature Phys. 4, 706 (2008).

[9] D. Sanvitto, F. Marchetti, M. Szymańska, G. Tosi, M. Baudisch, F. Laussy, D. Krizhanovskii, M. Skolnick, L. Marrucci, A. Lemaître, J. Bloch, C. Tejedor, and L. V. Na, Nature Phys. 6, 527 (2010).

[10] P. Cristofolini, A. Dreismann, G. Christmann, G. Franchetti, N. G. Berloff, P. Tsotsis, Z. Hatzopoulos, P. G. Savvidis, and J. J. Baumberg, Phys. Rev. Lett. 110, 186403 (2013).

[11] R. Y. Chiao and J. Boyce, Phys. Rev. A 60, 4114 (1999).

[12] E. L. Bolda, R. Y. Chiao, and W. H. Zurek, Phys. Rev. Lett. 86, 416 (2001).

[13] A. Tanzini and S. Sorella, Phys. Lett. A 263, 43 (1999).
[14] L. A. Lugiato and R. Lefever, Phys. Rev. Lett. 58, 2209 (1987).

[15] M. Brambilla, L. A. Lugiato, V. Penna, F. Prati, C. Tamm, and C. O. Weiss, Phys. Rev. A 43, 5114 (1991).

[16] K. Staliunas, Phys. Rev. A 48, 1573 (1993).

[17] D. Snoke and S. Girvin, J. Low Temp. Phys. 171, 1 (2013).

[18] L. A. Lugiato and C. Oldano, Phys. Rev. A 37, 3896 (1988).

[19] R. Boyd, Nonlinear optics, 3rd ed. (Academic Press, New York, 2008).

[20] J. Keeling and N. G. Berloff, Phys. Rev. Lett. 100, 250401 (2008).

[21] Z. Hadzibabic and J. Dalibard, Riv. Nuovo Cimento 34, 389 (2011).

[22] F. M. Marchetti, J. Keeling, M. H. Szymańska, and P. B. Littlewood, Phys. Rev. B 76, 115326 (2007).

[23] M. H. Szymańska, J. Keeling, and P. B. Littlewood, Phys. Rev. B 75, 195331 (2007).

[24] J. Keeling, P. R. Eastham, M. H. Szymanska, and P. B. Littlewood, Phys. Rev. B 72, 115320 (2005).

[25] I. Carusotto and C. Ciuti, Rev. Mod. Phys. 85, 299 (2013).

[26] M. H. Szymańska, J. Keeling, and P. B. Littlewood, Phys. Rev. Lett. 96, 230602 (2006).

[27] M. Wouters and I. Carusotto, Phys. Rev. A 76, 043807 (2007).

[28] P. O. Fedichev and G. V. Shlyapnikov, Phys. Rev. A 58, 3146 (1998).

[29] L. Pitaevskii and S. Stringari, Bose-Einstein Condensation (Oxford University Press, Oxford, 2003).

[30] Dye Lasers, edited by F. Schaefer (Springer-Verlag, Berlin, 1990).

[31] S. Delysse, J.-M. Nunzi, and C. Scala-Valero, Appl. Phys. B 66, 439 (1998) 